\title{
Dynamic reorganization of intrinsic functional networks in the mouse brain
}

Joanes Grandjean ${ }^{1}$, Maria Giulia Preti ${ }^{2,3}$, Thomas AW Bolton ${ }^{2,3}$, Michaela Buerge $^{4}$, Erich Seifritz ${ }^{5,6}$, Christopher R Pryce ${ }^{4,6}$, Dimitri Van De Ville ${ }^{2,3}$, Markus Rudin ${ }^{1,6,7}$

1 Institute for Biomedical Engineering, University and ETH Zurich, Zurich, Switzerland

2 Institute of Bioengineering, École Polytechnique Fédérale de Lausanne (EPFL), Lausanne, Switzerland

3 Department of Radiology and Medical Informatics, University of Geneva, Geneva, Switzerland 4 Preclinical Laboratory for Translational Research into Affective Disorders, DPPP, Psychiatric Hospital, University of Zurich, Zurich, Switzerland

5 Department of Psychiatry, Psychotherapy and Psychosomatics (DPPP), Psychiatric Hospital, University of Zurich, Zurich, Switzerland

6 Neuroscience Center Zurich, University of Zurich and ETH Zurich, Zurich, Switzerland

7 Institute of Pharmacology and Toxicology, University of Zurich, Zurich, Switzerland

\section{Corresponding author:}

Prof. Dr. Markus Rudin

Institute for Biomedical Engineering

University and ETH Zürich

\section{AIC-ETH HCI D426}

Vladimir-Prelog-Weg 4

$\mathrm{CH}-8093$ Zürich

Tel: +41(0)44633 7604

Fax: +41(0)446331187

e-mail: rudin@biomed.ee.ethz.ch 


\begin{abstract}
Functional connectivity (FC) derived from resting-state functional magnetic resonance imaging (rs$\mathrm{fMRI}$ ) allows for the integrative study of neuronal processes at a macroscopic level. The majority of studies to date have assumed stationary interactions between brain regions, without considering the dynamic aspects of network organization. Only recently has the latter received increased attention, predominantly in human studies. Applying dynamic FC (dFC) analysis to mice is attractive given the relative simplicity of the mouse brain and the possibility to explore mechanisms underlying network dynamics using pharmacological, environmental or genetic interventions. Therefore, we have evaluated the feasibility and research potential of mouse dFC using the interventions of social stress or anesthesia duration as two case-study examples. By combining a sliding-window correlation approach with dictionary learning, several dynamic functional states (dFS) with a complex organization were identified, exhibiting highly dynamic inter- and intra-modular interactions. Each dFS displayed a high degree of reproducibility upon changes in analytical parameters and across datasets. They fluctuated at different degrees as a function of anesthetic depth, and were sensitive indicators of pathology as shown for the chronic psychosocial stress mouse model of depression. Dynamic functional states are proposed to make a major contribution to information integration and processing in the healthy and diseased brain.
\end{abstract}

Keywords: mouse, fMRI, functional connectivity, dynamic functional states, dictionary learning 